\title{
Numerical post failure methods in multiphysical problems
}

\author{
Collin $\mathrm{F}_{.}^{*, * * *}$ - Levasseur S. ${ }^{*, * *}-$ Chambon $\mathbf{R}^{* *}$ \\ * Université de Liège \\ Institut de Mécanique et Génie Civil \\ Chemin des Chevreuils 1, 4000 Liège \\ Sart Tilman, Belgique \\ f.collin@ulg.ac.be \\ ${ }^{* *}$ Laboratoires 3S-R, Université Joseph Fourier, Grenoble \\ ${ }^{* * *}$ FRS-FNRS Fonds National de la Recherche Scientifique
}

RÉSUMÉ. La rupture dans les géomatériaux est souvent précédée par la formation de fines bandes de localisation des déformations. La formation de ces bandes de localisation est un processus non négligeable, étudié à la fois sur le plan expérimental et sur le plan théorique. Cet article résume les principaux phénomènes observés sur les processus localisés et propose quelques outils théoriques et numériques nécessaires à la caractérisation de ces processus de localisation. Afin de tenir compte des interactions entre les différentes phases des milieux poreux, une technique de régularisation basée sur des modèles de type second gradient est étendue aux couplages mutiphysiques.

ABSTRACT. Rupture in geomaterials is often preceded by a localization of the deformations within thin bands. The strain localization is thus an important process, which has been studied both experimentally and theoretically. This paper summaries main observations on localized phenomena and proposes theoretical and numerical tools to characterize localization processes. To deal with interactions occurring between the different phases of porous media, a regularization technique based on the second gradient model has been extended to multiphysic couplings.

MOTS-CLÉS : localisation des déformations, modélisation couplée, milieux enrichis, modèle second gradient.

KEYWORDS: strain localization, coupled modelling, enriched continuum, second gradient model.

Revue. Volume $\mathrm{X}-\mathrm{n}^{\circ} \mathrm{x} /$ année, pages 1 à $\mathrm{X}$ 


\section{Introduction}

In many Civil Engineering problems (in a broad sense), the rupture of the foundation soil may be the relevant phenomenon for the global stability of a structure. For geomaterials, experimental observations show clearly localized ruptures (Desrues et al., 1996). Some authors (Khoa et al., 2006) refer also to diffuse mode of failure (which means homogeneous failure in laboratory tests). In the first case, the phenomenon is preceded by progressive strain localization within thin bands, leading finally to rupture lines. It is thus very important to be able to model numerically the post localization behaviour of geomaterials, for applications like the evaluation of bearing capacity, of slope stability or of damage zone around a tunnel... Furthermore, soils and rocks are porous materials, where the porous volume is filled with one or several fluids (water, gas, oil ...). The general behaviour of the medium depends not only on the skeleton response (solid phase) to a given loading path, but also on the interactions occurring between the different phases of the medium. Capillary effects, temperature variations, chemical reactions induce specific behaviours, which have to be modelled by multiphysical constitutive laws. It has been shown that these latter laws share a same structure (See Collin et al., 2005). The numerical tools for the modelling of post failure problems have thus to be extended to this multiphysic context, to deal with applications related to nuclear waste disposal, concrete behaviour under severe loading... Then, new questions arise concerning the interactions between localization process and physical process (like liquid diffusion for example). The answer to these new questions can only be given by experimental evidences. The section 2 of this paper will summarized the main observations on localization phenomenon, coming from the experimental results. The section 3 is related to the theoretical tools devoted to localization process. The section 4 is the description of the coupled second gradient model, which is the regularization technique used for the application described in section 5. Some conclusions end up the paper in section 6 .

\section{Experimental evidences}

During the last 20 years, a lot of experimental work has been devoted to the study of strain localization in soils and rocks. These studies performed by Vardoulakis and co-workers (Vardoulakis et al., 1978, Han et al., 1991), Finno and co-workers (Finno et al., 1996, Finno et al., 1997) and Desrues and co-workers (Desrues, 1984, Desrues et al., 2004) among others have mainly focused on the purely mechanical problem (dry sample or globally drained at low strain rate) or on the hydromechanical problem (undrained or globally drained at fast strain rate). The study of the evolution of strain localization processes during the experiment needs for special techniques like stereophotogrammetry, 3D digital image correlation or $\mathrm{X}$-ray micro tomography (Lenoir et al., 2007). Thus, as in triaxial experiment (and more generally in axi-symmetric tests) the localization zone may remain more or 
less hidden inside the sample, most of the experimental campaigns on localization have been performed in biaxial apparatus, where this latter process is 'clearly' evidenced.

The main conclusions of the experimental works can be summarized as follow (Desrues, 2005):

- Strain localization in shear band mode can be observed in most laboratory tests leading to rupture in geomaterials,

- Complex localization patterns may be the result of specific geometrical or loading conditions,

- Well marked stress peaks in stress-strain curves can be considered as the signature of an established shear band system over the specimens (Figure $1)$.

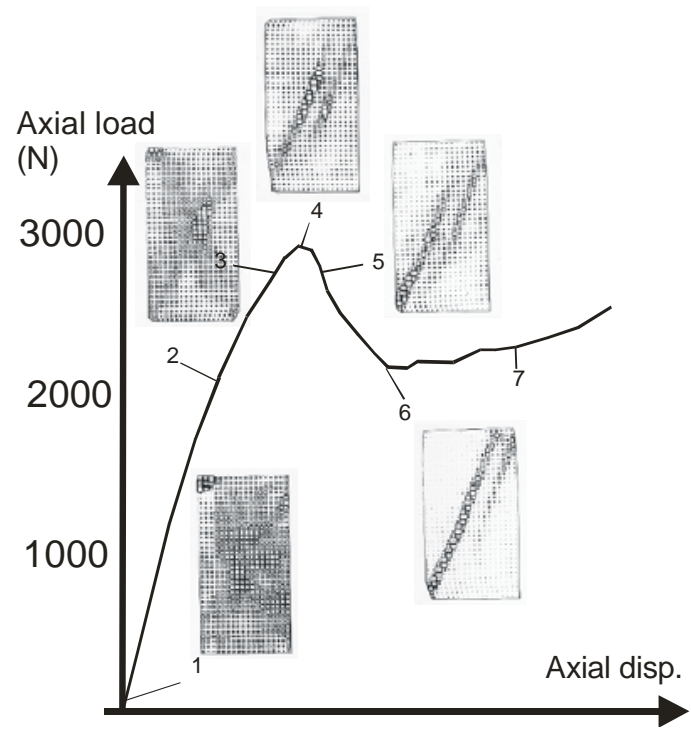

Figure 1. Drained biaxial test on Hostun sand [After Mokni et al., 1999]

Comparing to pure mechanical experiment (on dry sample), hydro-mechanical experiment introduces another physical phenomenon (i.e the fluid diffusion) that can influence the localization processes. This latter potential interaction depends of course on the (mechanical and diffusive) material properties, but also on the boundary conditions of the problem. For drained conditions (if the value of the permeability is sufficient low with respect of the velocity of the loading), the interactions are inexistent, as far as no overpressures are generated and the 
experimental observations are equivalent to those corresponding to dry conditions. For undrained conditions, overpressures are generated and some interactions exist between the two processes. It should be pointed out that, in the laboratory, the boundary conditions which can be prescribed correspond to globally undrained conditions and not to locally undrained ones. It means that, even in undrained conditions, some fluid flows can be created. The undrained experiments performed in Grenoble (Mokni et al., 1999; Roger et al., 1998) among others have shown some common features and have lead to the following observations:

- Plane strain undrained tests performed on loose and dense Hostun sand exhibit localization pattern.

- For dense (dilatant) sand specimen, localization is possible only when cavitation occurs in the pore fluid.

This last observation clearly evidences the interaction between the localization and the fluid diffusion, as far as the diffusion processes inhibits the localization before the cavitation within the pore volumes. Such phenomenon should thus be addressed both theoretically and numerically in a robust modelling of the rupture.

Experimental studies of the strain localisation in other multiphysic context (unsaturated conditions, thermo-mechanical, chemo-mechanical ...) are rather poor and there is clearly a need for further investigations. It is indeed important to know whether the temperature or the suction influence the occurrence of localization and the size of the process zone.

\section{Theoretical concepts}

The experimental results show that shear band formation can occur in geomaterials for many loading conditions. The constitutive laws devoted to the modelling of their behaviour have to be able to reproduce such phenomenon. The principal questions are thus the following: how could we know if the constitutive model can predict a strain localization mode and if this bifurcation occurs, how could we model it properly?

\subsection{Theoretical background}

Following the previous works by (Hadamard, 1903), (Hill, 1958) and (Mandel, 1966), Rice and co-workers (Rice, 1976, Rudnicki et al., 1975) have proposed the so-called Rice criterion, which analyses the stress state and investigates the possibility of a bifurcation by formation of a shear band in the stress and strain paths, for incrementally linear behaviour. The theoretical scheme of a shear band is presented on figure 2 . This criterion is based on a kinematical condition, a static 
condition and on the constitutive equation. The superscript 0 denotes variables outside the band and the superscript 1 denotes variables inside the band.
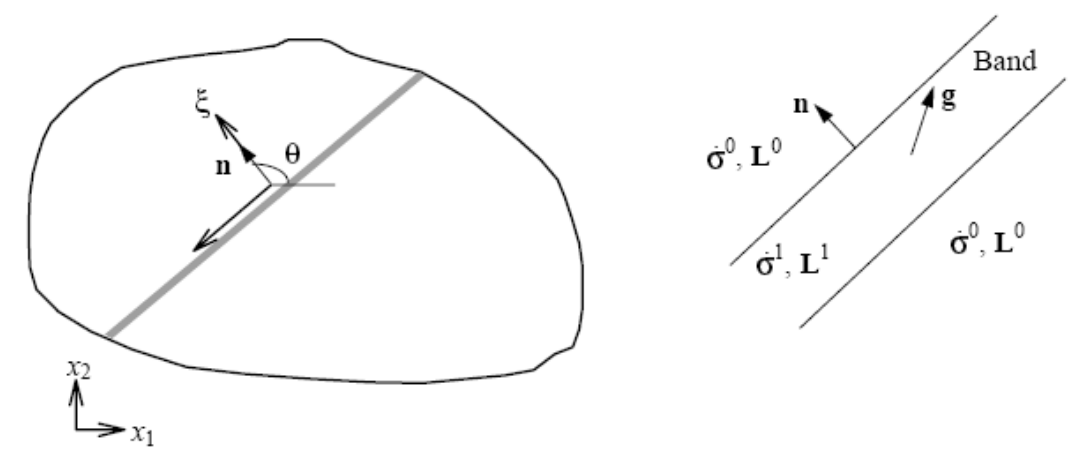

Figure 2. Theoretical scheme of a shear band

The static condition expresses the surface equilibrium at the interface between the inner and outer band:

$$
n\left(\dot{\sigma}^{1}-\dot{\sigma}^{0}\right)=0
$$

The kinematic condition expresses the strain jump across the band interface by a dilatant strain jump and a shear strain jump, but without any longitudinal strain jump

$$
L^{1}=L^{0}+g \otimes n
$$

where $L$ is the velocity gradient, $n$ is the vector normal to the band, and $g$ is a vector describing the band mode.

The third equation introduced in the Rice criterion is the constitutive law, which is generally speaking an incrementally non linear relationship. If it is assumed to be linear, one has then:

$$
\dot{\sigma}=C: L
$$

where $C$ is the assumed constitutive tangent tensor. If different constitutive tensors are assumed inside $C^{1}$ and outside $C^{0}$ the band, then introducing [3] into [1] and [2] gives a third order equation system in which the unknowns are the components of the $g$ vector.

$$
n\left(C^{1}:\left(L^{0}+g \otimes n\right)-C^{0}: L^{0}\right)=0
$$


The trivial solution $g=0$ is always possible but means that no shear band can appear. When it is assumed that $C^{\mathrm{l}}=C^{0}=C$, the condition for $g \neq 0$ is met if and only if the determinant of the acoustic tensor is less or equal to zero:

$$
\operatorname{det}(n C n) \leq 0
$$

This assumption is a strong one, often forgotten. However, in the case of a classical single-mechanism elastoplastic model, it has been proved that the previous criterion holds, provided $C$ is chosen as the constitutive tensor corresponding to plastic loading (Chambon, 1986).

These developments are valid for pure mechanical analysis. In a multiphysic context, Loret and co-workers (Loret et al., 1991) showed that for hydromechanical problems the condition of localization depends only on the drained properties of the medium. Nevertheless, it should be emphasized that in coupled problems much more complex localization pattern can be obtained, at least theoretically (figure 3; Vardoulakis, 1996, assuming an incrementally linear constitutive equation).

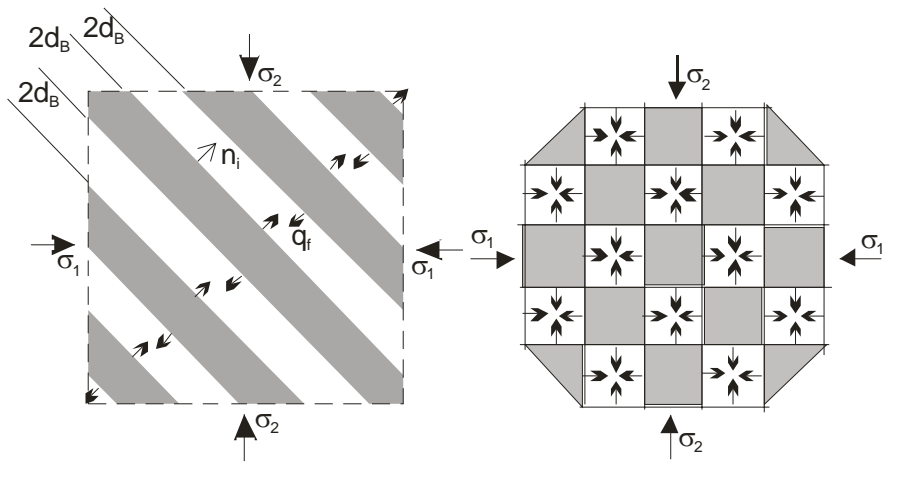

Figure 3. Shear banding mode in water-saturated sand (Vardoulakis, 1996)

\subsection{Regularization techniques}

Rupture in localized mode is observed experimentally; theoretical tools permit to characterize it when the conditions of occurrence of such phenomenon are met. The further step is to define a correct and robust modelling of the strain localization leading finally to the rupture. However, it is well known that classical finite element models suffer of a pathological problem of mesh dependency for strain localisation modelling. This is due to properties of the underlying mathematical problem. In order to put in evidence this deficiency of classical tools, let us consider a very simple one-dimensional problem of a constant section bar under uniaxial tension (Figure 4), made of a material with a very simple constitutive law (Figure 5-a). This law may be the result of an elastic damage model or a strain softening elastoplastic 
model. It should be pointed out that the following discussion is also valid for a material layer under shear (replacing normal stress by shear stress).

When submitted to an axial displacement, the load-displacement curve remains linear up to the stress state reaches the yield stress $f_{y}$. After this point, the axial stress decreases but it has to remain homogeneous all along the bar according the static equilibrium condition. For each point of the bar, two options exist: either the softening loading or the elastic unloading. Let us define $\alpha$ as the ratio of the length $L_{s}$ (where we have softening loading) over $L$. For $\alpha=1$, the load will decrease linearly down to zero for $\Delta=L \varepsilon_{r}$. For a limit case $(\alpha=0)$, the load will decrease linearly down to zero at the origin without energy consummation (Figure 5-b). A priori, with classical numerical model, the length $L_{s}$ is undetermined and, as far as the problem is ill-posed, we observe a mesh-dependency of the response of the model. For an algorithm finding the most localized solution, $L_{s}$ will be equal to the size of the smallest element: if the number of element is increasing, the ratio $\alpha$ will tend to zero! The situation is even worse, when considering the complete initial boundary value problem (see Kotronis et al., 2008).

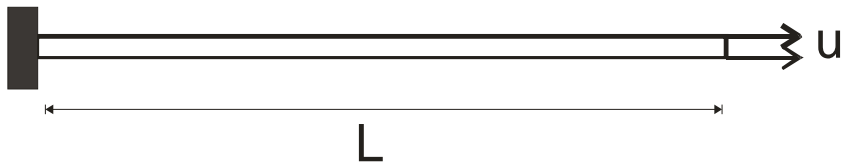

Figure 4. Constant section bar under uni-axial tension

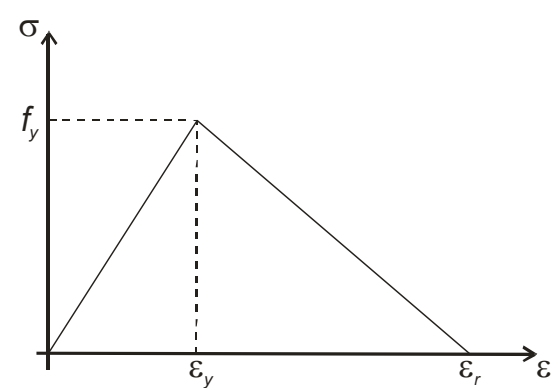

(a) : Local constitutive law

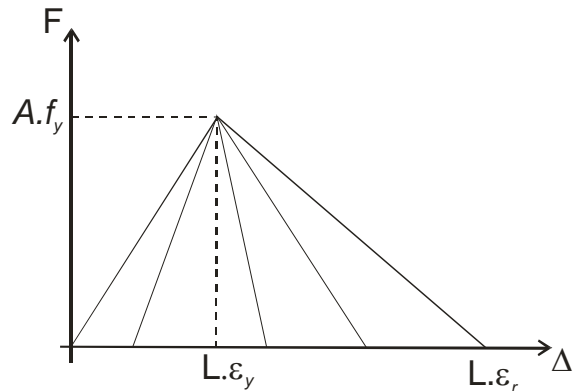

(b) : Global response of the bar

Figure 5. Local and global behaviour of the bar

This simple example evidences the need of an internal length scale for a correct modelling of the post peak behaviour. Several approaches have been proposed to introduce such internal length scale in the problem. We can distinguish two categories of regularization techniques: 
- Enrichment of the constitutive law

The theory introduces an internal length scale at the level of the constitutive model. The advanced analysis of localization phenomena has shown that constitutive equations with internal length are necessary to properly model the experimental results involving some localized patterns: see for instance the pioneering works of Aifantis (1984), which introduced gradient of internal variables, or the works of Bazant, Pijaudier-Cabot and co-workers (Bazant et al., 1984, Pijaudier-Cabot et al., 1987), which proposed a family of constitutive models derived from non local damage theory in which a non local internal variable is used instead of the local one. This latter theory has also generated the implicit gradient models (Peerlings et al., 1996), where the non local internal variable is an additional unknown and solution of the Helmoltz differential equation.

\section{- $\quad$ Enrichment of the kinematics}

The theories based on an enhancement of the kinematics itself can be traced back to the pioneering works of (Toupin, 1962), (Mindlin, 1964) and (Germain, 1973). Here we consider the materials with microstructure as defined by (Mindlin, 1964) and (Germain, 1973). Adding some mathematical constraints to the more general materials with microstructure yields a large set of models. Among all these models, the more ancient and famous one is the Cosserat model (Cosserat and Cosserat, 1909). In the following, we will mainly use the second gradient model developed in Grenoble (Chambon et al., 1998 and 2001), but the obtained conclusions can be generalized to the other regularization techniques.

The extension of these regularization techniques to multiphasic materials and multi-physic problems leads to the question of interactions between the physical problems and the localization process. Loret and co-workers have been shown that diffusion equation (fluid or thermal) introduces an internal length scale (Loret et al., 1991). Zhao, Schrefler and co-workers (Zhang et al., 1999, Zhang et al., 2001) have studied the potential interaction between the different lengths. They showed that the interaction between the different phenomena can appear for a given range of parameters, nevertheless a robust mechanical model is still needed even for coupled problems.

\section{Numerical modelling}

Among the different regularization techniques, we propose in the following to use the second gradient model (Chambon et al., 1998 and 2001). The second grade model is extended to biphasic medium (Collin et al., 2006) in order to show the possible interaction between the internal lengths introduced by the model and the diffusion process. In this model, the porous medium is considered as the superposition of several continua (Coussy, 1995): the solid skeleton (grains 
assembly) and the fluid phases (water, air, oil...). Based on averaging theories (Hassanizadeh and Gray, 1979a, 1979b), (Lewis and Schrefler, 2000) proposed the governing equations for the full dynamic behaviour of a partially saturated porous medium. Hereafter these equations are restricted for quasi-static problem in unsaturated conditions, under Richard's assumptions (constant air pressure). This assumption may be irrelevant in the particular case of low and ultra low permeable porous media. For this study, isothermal condition and incompressible solid grains are assumed. The unknowns of the mechanical and the flow problems are respectively the displacements $u_{i}$ and the pore water pressure $p_{w}$ (possibly negative in unsaturated case). In the following developments, the balance equations are written in the current solid configuration denoted $\Omega^{t}$ (updated Lagrangian formulation).

\subsection{Balance of momentum equation}

In the mixture balance of momentum equation, the interaction forces between fluid phases and grain skeleton cancels. In a weak form (virtual work principle), this equation reads for any kinematically admissible virtual displacement field $u_{i}^{*}$ :

$$
\int_{\Omega^{t}} \sigma_{i j}^{t} \varepsilon_{i j}^{*} d \Omega^{t}=\int_{\Omega^{\prime}}\left(\rho_{s}\left(1-\phi^{t}\right)+S_{r, w}^{t} \rho_{w}^{t} \phi^{t}\right) g_{i} u_{i}^{*} d \Omega^{t}+\int_{\Gamma_{\sigma}^{t}} \bar{t}_{i}^{t} u_{i}^{*} d \Gamma^{t}
$$

where $\varepsilon_{i j}^{*}=0.5\left(\left(\partial u_{i}^{*} / \partial x_{j}^{t}\right)+\left(\partial u_{j}^{*} / \partial x_{i}^{t}\right)\right)$ is the kinematically admissible virtual strain field, $\phi^{t}$ is the porosity defined as $\phi^{t}=\Omega^{v, t} / \Omega^{t}$ where $\Omega^{t}$ is the current volume of a given mass of skeleton and $\Omega^{v, t}$ the corresponding porous volume, $\rho_{s}$ is the solid grain density, $S_{r, w}^{t}$ is the water relative saturation, $\rho_{w}^{t}$ is the water density, $g_{i}$ is the gravity acceleration and $\Gamma_{\sigma}^{t}$ is the part of the boundary where tractions $\bar{t}_{i}^{t}$ are known.

The total stress $\sigma_{i j}^{t}$ is defined as a function of the kinematics. Here we assume first that the Bishop's definition of effective stress holds (Nuth and Laloui, 2008):

$$
\sigma_{i j}^{t}=\sigma_{i j}^{t}-S_{r, w}^{t} p_{w}^{t} \delta_{i j}
$$

with $\sigma_{i j}^{t}$ the effective stress, $p_{w}^{t}$ the pore water pressure and $\delta_{i j}$ the Kronecker symbol.

\subsection{Mass balance equation}

The water mass balance equation reads in a weak form: 


$$
\int_{\Omega^{\prime}}\left(\dot{M}^{t} p_{w}^{*}-m_{i}^{t} \frac{\partial p_{w}^{*}}{\partial x_{i}^{t}}\right) d \Omega^{t}=\int_{\Omega^{\prime}} Q^{t} p_{w}^{*} d \Omega^{t}-\int_{\Gamma_{q}^{\prime}} \bar{q}^{t} p_{w}^{*} d \Gamma^{t}
$$

where $p_{w}^{*}$ is the virtual pore water pressure field, $Q^{t}$ is a sink term and $\Gamma_{a}^{t}$ is the part of the boundary where the input water mass per unit area $\bar{q}^{t}$ is prescribed. $M^{t}$ and $m_{i}^{t}$ are respectively the mass of the water inside the current configuration of the skeleton $\Omega^{t}$ and the mass flow. They are defined hereafter respectively in Equation [11] and Equation [9].

Water mass balance equation (Equation 8) has to hold for any time $t$, the virtual quantities in this equation being dependant on the history of boundary conditions and on time $t$.

The mass flow $m_{i}^{t}$ is defined as follows:

$$
m_{i}^{t}=-\rho_{w}^{t} \frac{\kappa k_{r, w}^{t}}{\mu_{w}}\left(\frac{\partial p_{w}^{t}}{\partial x_{i}^{t}}+\rho_{w}^{t} g_{i}\right)
$$

where $\kappa$ is the intrinsic permeability, $k_{r, w}^{t}$ is water relative permeability and $\mu_{w}$ is the water dynamic viscosity.

The compressible fluid is assumed to respect the following relationship (Lewis and Schrefler, 2000). This predicts an increase of water density as a function of the pore water pressure, defining $\chi_{w}$ as the water bulk modulus:

$$
\dot{\rho}_{w}^{t}=\frac{\rho_{w}^{t}}{\chi_{w}} \dot{p}_{w}^{t}
$$

If the grains are assumed to be incompressible (which means $\rho_{s}$ is constant), the time derivative of the water mass is obtained directly by using Equation [10] and mass balance equation for the solid phase. This yields for a unit mixture volume:

$$
\dot{M}^{t}=\rho_{w}^{t}\left[\frac{\dot{p}^{t}}{\chi_{w}} S_{r, w}^{t} \phi^{t}+\dot{S}_{r, w}^{t} \phi^{t}+S_{r, w}^{t} \frac{\dot{\Omega}^{t}}{\Omega^{t}}\right]
$$

\subsection{Local second gradient model for monophasic medium}

Let us recall first the governing equations of a monophasic medium with microstructure. This kind of models can be traced back to the pioneering work of the Cosserat brothers (Cosserat and Cosserat, 1909), (Mindlin, 1964) and (Germain, 1973). In the framework of microstructure continuum theory, a microkinematic gradient field $v_{i j}$ is introduced to describe strain and rotation at the microscale. With respect to classical continuum mechanics, additional terms are then added in the 
internal virtual work of a given body (Germain, 1973). The following expression holds for any virtual quantities:

$$
W_{i}^{*}=\int_{\Omega^{\prime}}\left(\sigma_{i j}^{t} \frac{\partial u_{i}^{*}}{\partial x_{j}^{t}}+\tau_{i j}^{t}\left(v_{i j}^{*}-F_{i j}^{*}\right)+\Sigma_{i j k}^{t} h_{i j k}^{*}\right) d \Omega^{t}
$$

where $F_{i j}^{*}$ is the virtual macrodeformation gradient, $v_{i j}^{*}$ is the virtual microkinematic gradient, $\tau_{i j}^{t}$ called microstress is an additive stress associated to the microstructure, $h_{i j k}^{*}$ is the virtual microsecond gradient and $\Sigma_{i j k}^{t}$ is the double stress dual of $h_{i j k}^{*}$.

In the local second gradient model used in the following, an assumption is added: the micro kinematic gradient is equal to the macro-deformation gradient

$$
v_{i j}=F_{i j}
$$

As a consequence: $v_{i j}^{*}=F_{i j}^{*}$. Finally, the principle of virtual work can be rewritten as follows:

$$
W_{i}^{*}=\int_{\Omega^{t}}\left(\sigma_{i j}^{t} \frac{\partial u_{i}^{*}}{\partial x_{j}^{t}}+\Sigma_{i j k}^{t} \frac{\partial^{2} u_{i}^{*}}{\partial x_{j}^{t} \partial x_{k}^{t}}\right) d \Omega^{t}
$$

Assuming that the boundary $\Omega^{t}$ is regular, the external virtual work $W_{e}^{*}$ can be defined as follows:

$$
W_{e}^{*}=\int_{\Omega^{\prime}} \rho g_{i} u_{i}^{*} d \Omega^{t}+\int_{\Gamma_{\sigma}^{\prime}}\left(\bar{t}_{i} u_{i}^{*}+\bar{T}_{i} D u_{i}^{*}\right) d \Gamma^{t}
$$

where $\bar{t}_{i}$ is the external (classical) forces per unit area and $\bar{T}_{i}$ an additional external (double) force per unit area, both applied on a part $\Gamma_{\sigma}^{t}$ of the boundary of $\Omega^{t} . D$ denotes the normal derivative of any quantity $q,\left(D q=\left(\partial q / \partial x_{k}\right) n_{k}\right)$. To the authors' knowledge, the additional boundary condition (on $\bar{T}_{i}$ ) allows to produce solutions with boundary layers.

One of the advantages of local second gradient is the fact that constitutive equations remain local and therefore it is straightforward to formulate a second gradient extension of any classical models. The previous virtual work equation of second gradient models can of course be used in a finite element code. However, this equation needs the use of $C^{l}$ functions for the displacement field as second derivatives of the displacement are involved. In order to avoid such functions in the virtual work principle [14], the equalities between $v_{i j}^{*}$ and $F_{i j}^{*}$ and between $v_{i j}$ and 
$F_{i j}$ are introduced through a field of Lagrange multipliers $\lambda_{\mathrm{ij}}$ related to a weak form of the constraint [13] (see Chambon et al., 1998):

$$
\begin{gathered}
\int_{\Omega^{\prime}}\left(\sigma_{i j}^{t} \frac{\partial u_{i}^{*}}{\partial x_{j}^{t}}+\sum_{i j k}^{t} \frac{\partial v_{i j}^{*}}{\partial x_{k}^{t}}\right) d \Omega^{t}-\int_{\Omega^{\prime}} \lambda_{i j}^{t}\left(\frac{\partial u_{i}^{*}}{\partial x_{j}^{t}}-v_{i j}^{*}\right) d \Omega^{t}-W_{e}^{*}=0 \\
\int_{\Omega^{\prime}} \lambda_{i j}^{*}\left(\frac{\partial u_{i}^{t}}{\partial x_{j}^{t}}-v_{i j}^{t}\right) d \Omega^{t}=0
\end{gathered}
$$

\subsection{Local second gradient coupled model}

Starting from Equations [6] and [8] of the coupled problem in classical poromechanics, microstructure effects can be introduced in the momentum balance equation by adding microkinematic gradient terms, under the previous assumptions $\left(v_{i j}^{*}=F_{i j}^{*}, v_{i j}=F_{i j}\right)$. Assuming that pore fluid has no influence at the microstructure level, microkinematic gradient are not generated by pore pressure variations. This latter hypothesis follows the ideas of Ehlers (Ehlers et al., 1998) concerning a Cosserat model for biphasic medium. Cosserat model (Cosserat and Cosserat, 1909) is in fact a particular case of microstructured medium.

According to the previous assumptions, we have the following governing equations:

$$
\begin{gathered}
\int_{\Omega^{\prime}}\left(\sigma_{i j}^{t} \frac{\partial u_{i}^{*}}{\partial x_{j}^{t}}+\Sigma_{i j k}^{t} \frac{\partial^{2} u_{i}^{*}}{\partial x_{j}^{t} \partial x_{k}^{t}}\right) d \Omega^{t}=\int_{\Omega^{\prime}} \rho^{m i x, t} g_{i} u_{i}^{*} d \Omega^{t}+\int_{\Gamma_{\sigma}^{\prime}}\left(\bar{t}_{i}^{*} u_{i}^{*}+\bar{T}_{i} D u_{i}^{*}\right) d \Gamma^{t} \\
\int_{\Omega^{\prime}}\left(\dot{M}^{t} p_{w}^{*}-m_{i}^{t} \frac{\partial p_{w}^{*}}{\partial x_{i}^{t}}\right) d \Omega^{t}=\int_{\Omega^{\prime}} Q^{t} p_{w}^{*} d \Omega^{t}-\int_{\Gamma_{q}^{\prime}} \bar{q}^{t} p_{w}^{*} d \Gamma^{t}
\end{gathered}
$$

where $\rho^{m i x, t}$ is the mass density of the mixture $\left(\rho^{m i x, t}=\rho^{s}\left(1-\phi^{t}\right)+\rho^{w, t} \phi^{t}\right)$. Introducing Lagrange multiplier fields (like in the previous section), the governing equations of the local second gradient coupled problem are then the following:

$$
\begin{gathered}
\int_{\Omega^{\prime}}\left(\sigma_{i j}^{t} \frac{\partial u_{i}^{*}}{\partial x_{j}^{t}}+\Sigma_{i j k}^{t} \frac{\partial v_{i j}^{*}}{\partial x_{k}^{t}}\right) d \Omega^{t}-\int_{\Omega^{\prime}} \lambda_{i j}^{t}\left(\frac{\partial u_{i}^{*}}{\partial x_{j}^{t}}-v_{i j}^{*}\right) d \Omega^{t}-W_{e}^{*}=0 \\
\int_{\Omega^{\prime}} \lambda_{i j}^{*}\left(\frac{\partial u_{i}^{t}}{\partial x_{j}^{t}}-v_{i j}^{t}\right) d \Omega^{t}=0
\end{gathered}
$$




$$
\int_{\Omega^{\prime}}\left(\dot{M}^{t} p_{w}^{*}-m_{i}^{t} \frac{\partial p_{w}^{*}}{\partial x_{i}^{t}}\right) d \Omega^{t}=\int_{\Omega^{\prime}} Q^{t} p_{w}^{*} d \Omega^{t}-\int_{\Gamma_{q}^{t}} \bar{q}^{t} p_{w}^{*} d \Gamma^{t}
$$

In the present work, the stress tensor $\sigma_{i j}$ in Equation [20] is a total stress defined according to Terzaghi's postulate [7], on the contrary the double stress $\Sigma_{i j k}$ has no link with the pore pressure.

Equations [20]-[22] have to hold for any time $t$, the virtual quantities in these equations being dependant on the history of boundary conditions and then on time $t$. Moreover the constitutive equations also have to hold for any time $t$.

\subsection{Local second gradient model in a multiphysic context}

The procedure to extend local second gradient model to other multiphysical context is more or less the same: additional balance equations appear to model the other processes. The main issue is not a numerical one but rather a physical one. What are the possible interactions between the second gradient model and the thermal diffusion, the suction or the chemical reaction? These questions should be first addressed by the experimental campaign. Concerning the internal length introduced by the second gradient model, it comes as the ratio of two constitutive moduli (the one related to the second gradient constitutive law and the one related to the classical law). Considering that the classical constitutive moduli are influenced by the different processes (chemical, thermal, suction ...), the conclusions should be that the internal length scale should be modified by these latter processes (under the condition that second gradient law is not influenced by the processes). This should be again confirmed by some experimental evidences!

\section{Applications}

The following example exhibits the interest of using the theoretical tools presented but points out also their limitations. We propose to study a typical problem of nuclear waste disposal: the excavation of a storage gallery. It is proposed to model the excavation process with a very simple strain-softening constitutive law able to reproduce the main phenomena appearing during tunnelling: the progressive decrease of material strength during loading and the strain localisation. This coupled modelling is a benchmark exercise proposed by the GDRMomas and organized by EDF-CEA (Chavant et al., 2005).

\subsection{Definition of the problem}

The proposed constitutive law is an elasto-plastic strain-softening model. The yield criterion is a Drucker-Prager model given by the following equation: 


$$
F \equiv \sqrt{\frac{3}{2}} I I_{\sigma^{*}}+m\left(I_{\sigma}-\frac{3 c}{\tan \phi}\right)=0
$$

where $I I_{\sigma^{*}}$ is the second deviatoric stress invariant, $I_{\sigma}$ is the first stress invariant, $\phi$ is the friction angle, parameter $m$ is a function of $\phi: m=2 \sin (\phi) /(3-\sin (\phi))$, the cohesion $c=c_{0} f\left(\gamma^{p}\right)$ is the softening variable, $c_{0}$ is the initial cohesion and $\gamma^{p}$ is the equivalent deviatoric plastic deformation.

A cylindrical unsupported cavity of $3 \mathrm{~m}$ diameter is located in an homogeneous low permeability formation. The initial state of stress and pore pressure is the following:

$$
\begin{aligned}
& \sigma_{x x}^{\prime}=\sigma_{z z}^{\prime}=-7.74 \mathrm{MPa} \\
& \sigma_{y y}^{\prime}=-11.64 \mathrm{MPa} \\
& p_{w}=4.7 \mathrm{MPa}
\end{aligned}
$$

The excavation process is modelled by decreasing both radial total stress and the pore pressure at the cavity wall:

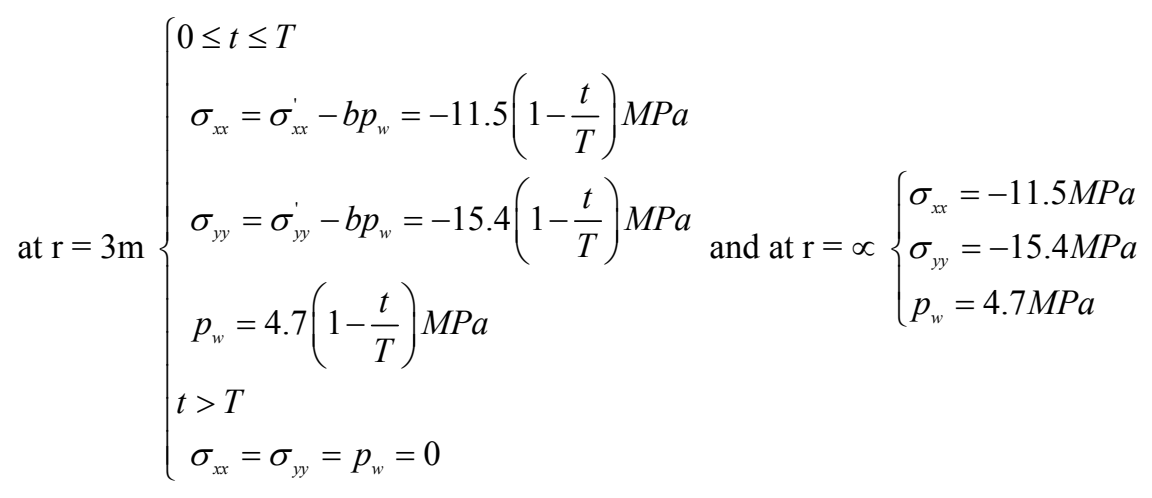

The excavation duration $T$ is equal to $1.5 \mathrm{Ms}$ (about 17 days) and the final modelling time is $300 \mathrm{Ms}$ (about 9.5 years).

\subsection{Constitutive law and Rice criterion}

As seen in section 3.1, the Rice criterion is relevant for a single mechanism classical elastoplastic model. In this case, the Rice criterion can be transformed in a fourth order equation in $\tan (\theta)=z$, with $\theta$ being the angle between $n$ and the $\mathrm{x}$-axis, and $a_{\mathrm{i}}$ being real parameters depending on the constitutive moduli:

$$
\left(a_{1} z^{4}+a_{2} z^{3}+a_{3} z^{2}+a_{4} z+a_{5}\right)=0
$$


At the beginning of loading, this equation does not have any real solution. After some load steps, the first real solution is a double one (Wang, 1993), which then gives two bifurcation directions.

In order to emphasize this theoretical concept with the proposed constitutive law, the homogeneous response of a drained biaxial experiment is first studied to observe when the Rice condition is met (Figure 6).

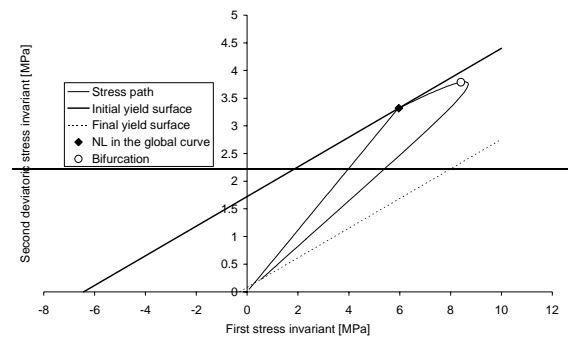

(a) : Local constitutive law

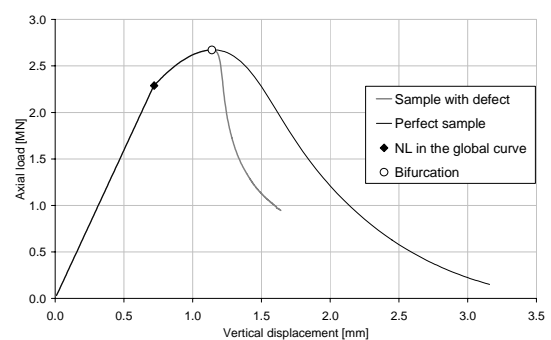

(b) : Global response of the bar

Figure 6. Biaxial test response of the constitutive law

Figure 7 presents the evolution of the Rice criterion as a function of the loading history. When the behaviour is elastic, the criterion exhibits only one minimum and is always positive. The behaviour becoming elastoplastic, the criterion exhibits two extrema but remains positive up to a loading level, for which two symmetric directions of bifurcation are predicted. The loading path going on, a fan of directions exists, where the Rice criterion is met. 


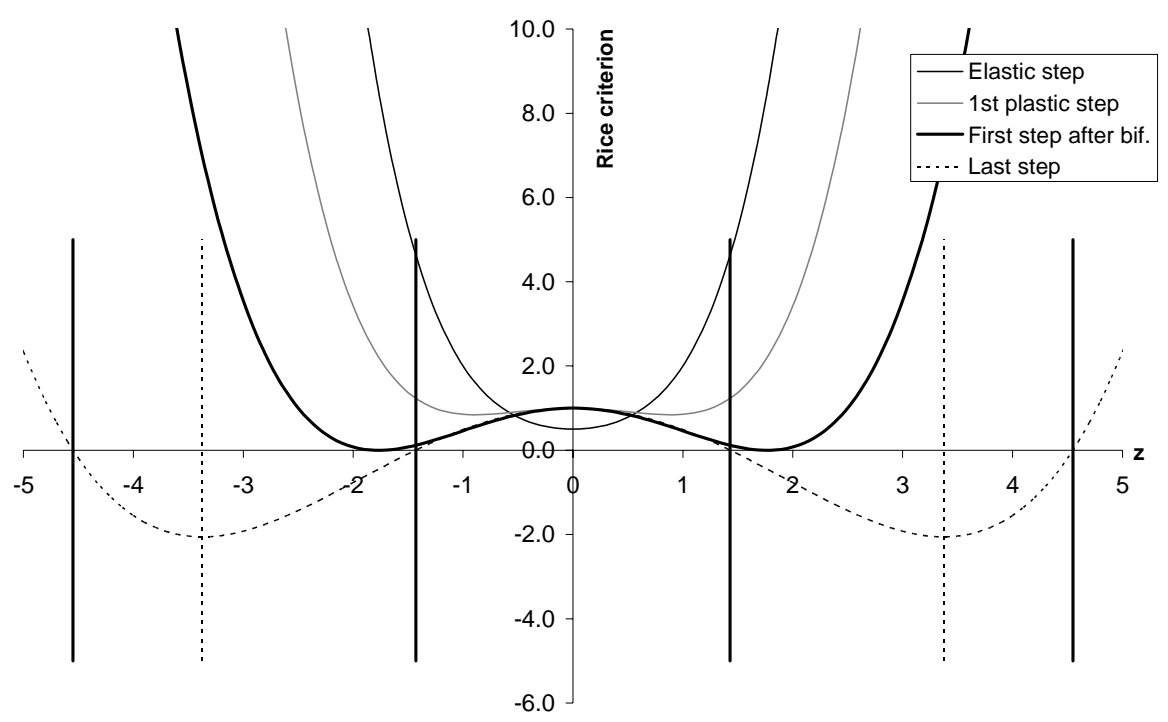

Figure 7. Rice criterion (as a function of z) for several loading steps

The numerical modelling of a perfect sample remains homogeneous even if the Rice criterion is met. In the reality, geomaterial samples have some heterogeneity, leading to localization processes. In order to reproduce numerically these processes, it is possible to introduce some defect (soft element, parasite force ...) in order to force the occurrence of localization (Figure 8). However, even in a perfect sample, it is possible to find localized solutions, using special techniques (See Chambon et al., 2001). 

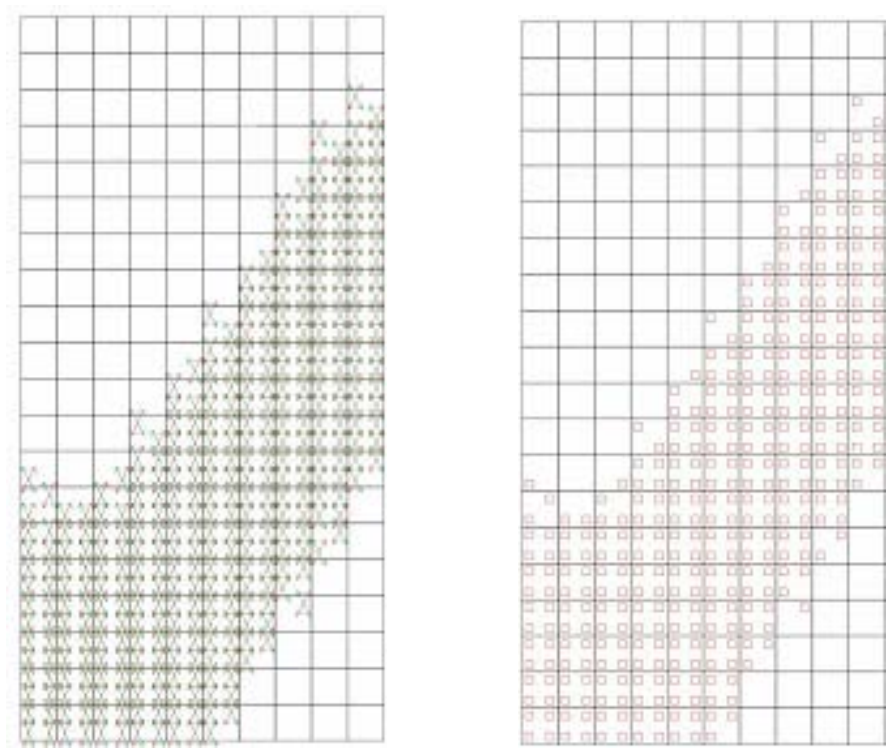

Figure 8. Localized solution for non-perfect sample

\subsection{Model predictions for the excavation problem}

During the excavation, the behaviour of the material becomes plastic near the tunnel and permanent strains are created. The yield criterion is first met in the direction where the orthoradial stress is the major principal stress. Dilatancy effects are evidenced on Figure 9-a, where the pore pressure becomes negative at the end of the tunnelling. Then, during the remaining modelling time, the pore pressure increases progressively. Figure 9-b shows the radial displacement curve: during the tunnelling phase, cavity convergence remains around $1,5 \mathrm{~cm}$. Most of the convergence appears later and reaches $22,6 \mathrm{~cm}$. Indeed, negative pore pressure allows a additional cohesive strength; this effect is maximum after 1,5 Ms and decreases progressively still $300 \mathrm{Ms}$.

Shear band localization is also influenced by the hydro-mechanical coupling. Indeed, at the end of excavation, there is no clear localization patterning even if the Rice's criterion is verified. After that phase, pore pressure increases progressively and a patterning is gradually created. Figure 10-a presents a map of the equivalent strains where the different shear bands clearly appear. The Vilotte's indicator is shown in Figure 10-b; this indicator evidences the strain activity of the shear band. One can see here that only the external shear band is active at the end of the simulation and that a chip is finally created. 


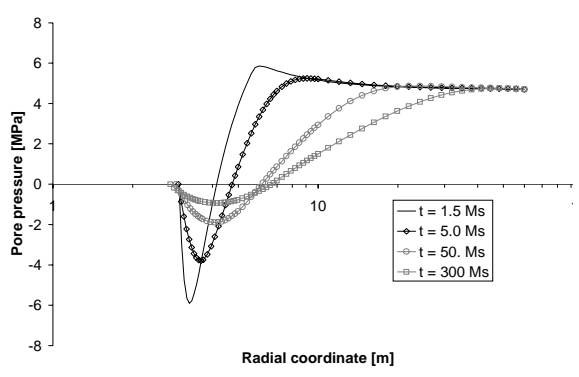

(a)

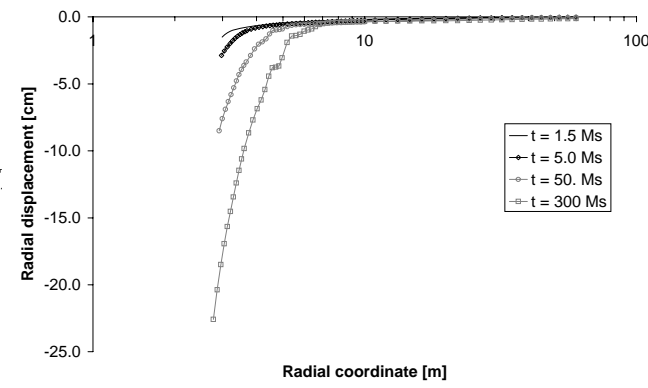

(b)

Figure 9. Pore pressure and displacement curve during coupled excavation

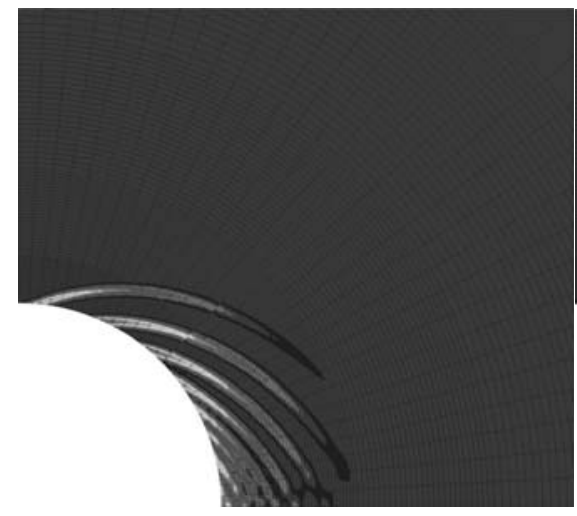

(a)

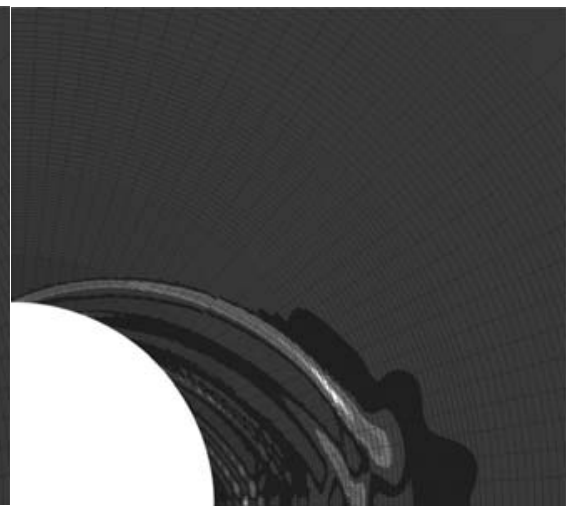

(b)

Figure 10. Equivalent strain and Vilotte's indicator for active shear band $(t=$ 300Ms) - Classical medium

These results (Figure 10) have been obtained with classical finite elements and are thus mesh dependent. We propose now to perform the same modelling with coupled second gradient model. We see (Figure 11) that the results are now independent of the mesh size. Regularization techniques are a solution for this problem but it should be pointed out that these theories do not restore the uniqueness of the solution. 

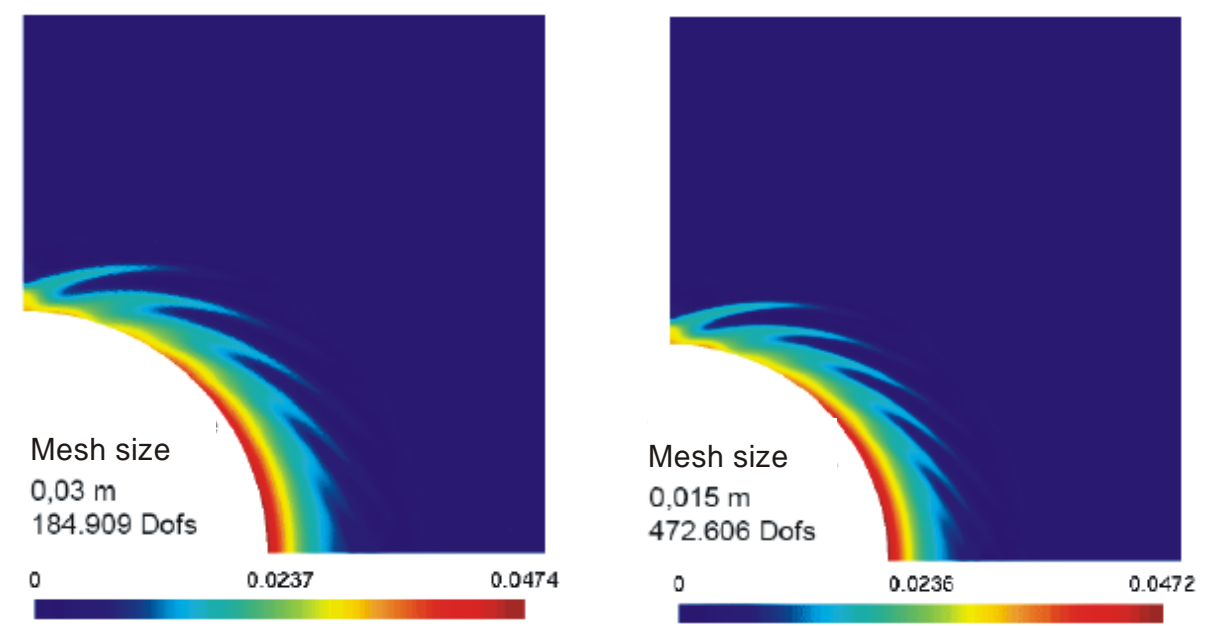

Figure 11. Equivalent strain for two mesh sizes with second gradient dilation model (Fernandes, 2009)

\section{Conclusions}

Rupture in geomaterials is often preceded by a localization of the deformations within thin bands. The strain localization is thus an important process, which has been studied both experimentally and theoretically. The developments of geomechanics in the field of coupled multiphysic processes impose the study of the strain localization to these new conditions. Interactions between the different processes can indeed occur.

The numerical modelling of shear bands with classical finite element suffers of a mesh dependency problem. An internal length scale has to be introduced in the problem. Among the different regularization techniques, we propose a second gradient coupled model for an application to gallery excavation. It has been shown that the model regularizes the solution but does not restore the uniqueness (see Sieffert et al., 2009). The extension of such theories to other multiphysic context is more an experimental problem than a numerical one. Experiments still have to exhibit the influence of temperature, suction or chemical concentration on the occurrence and the thickness of the strain localization! 


\section{Acknowledgment}

The authors are grateful to FNRS and EU (under TIMODAZ project) for their financial support.

\section{References}

Aifantis E.C., "On the microstructural origin of certain inelastic models", Journal of Engineering Materials and Technology, ASME, vol. 106, 1984, p. 326-330.

Bazant Z.P., Belytschko T.B., Chang T.P., "Continuum model for strain softening”, Journal of Engineering Mechanics, ASCE, vol. 110, 1984, p. 1666-1692.

Chambon R. "Bifurcation and shear band localization an approach for incrementally non linear constitutive equations", Journal de mécanique théorique et Appliquée, vol. 5, n 2 , 1986, p. $277-298$

Chambon R., Caillerie D., El Hassan N., "One-dimensional localisation studied with a second grade model”, Eur. J. Mech. A/Solids, vol. 17, n 4, 1998, p. 637-656.

Chambon R., Caillerie D., Matsushima T., "Plastic continuum with microstructure, local second gradient theories for geomaterials : localization studies", International Journal of Solids and Structures, vol. 38, 2001, p. 8503-8527.

Chambon R., Crochepeyre S., Charlier R., "An algorithm and a method to search bifurcation points in non-linear problems", International Journal for Numerical Methods in Engineering, vol. 51, 2001, p. 315-332.

Chavant C., Fernandes R., "Evaluating the reliability of hydro-mechanical simulation : a benchmark of numerical techniques carried out by Research Group of MoMas", $2^{\text {nd }}$ International Meeting Clays in Natural \& Engineered Barriers for Radioactive Waste Confinement, Tours, 14-18 March 2005, pp 249-250.

Collin F., Laloui L., Charlier R., "Unified approach of coupled constitutive laws", ALERT Autumn School 2005, Revue Européenne de Génie Civil, vol. 9, n 5-6, 2005, p. 713-724.

Collin F., Chambon R., Charlier R., "A finite element method for poro mechanical modelling of geotechnical problems using local second gradient models", International Journal for Numerical Methods in Engineering, vol. 65, $\mathrm{n}^{\circ} 11,2006$, p. 1749-1772.

Cosserat E., Cosserat F., Théorie des Corps Déformables, Paris, Hermann, 1909.

Coussy O., Mechanics of Porous Continua, London, Wiley, 1995.

Desrues J., La localisation de la déformation dans les matériaux granulaires, Thèse de doctorat es Sciences, Institut de Mécanique de Grenoble, 1984.

Desrues J., Chambon R., Mokni M., Mazerolles F., "Void ratio evolution inside shear bands in triaxial sand specimens studied by computed tomography", Géotechnique, vol. 46, $n^{\circ} 7$, 1996, p. 529-546. 
Desrues J., Viggiani G., "Strain localization in sand: an overview of the experimental results obtaines in Grenoble using stereophotogrammetry", International Journal for Numerical and Analytical Methods in Geomechanics, vol. 28, n 4, 2004, p. 279-321.

Desrues J., "Hydro-mechanical coupling and strain localization in saturated porous media", ALERT Autumn School 2005, Revue Européenne de Génie Civil, vol. 9, n 5-6, 2005, p. 619-634.

Ehlers W., Volk W., "On theoretical and numerical methods in the theory of porous media based on polar and non-polar elasto-plastic solid materials", International Journal of Solids and Structures, vol. 35, 1998, p. 4597- 4617.

Fernandes R., Modélisation numérique objective des problèmes hydromécaniques couplés dans le cas des géomatériaux, Thèse de doctorat, UJF Grenoble, 2009.

Finno R., Harris W., Mooney M., Viggiani G., "Strain localization and undrained steady state of sands", Journal of Geotechnical Engineering ASCE, vol. 122, nº6, 1996, p. 462-473.

Finno R., Harris W., Mooney M., Viggiani G., "Shear bands in plane strain compression of loose sand”, Géotechnique, vol. 47, n¹, 1997, p. 149-165.

Germain P., "The method of virtual power in continuum mechanics. Part 2 Microstructure", SIAM J. Appl. Math., vol. 25, 1973, p. 556-575.

Hadamar J., Leçon sur la propagation des ondes et les équations de l'hydrodynamique, Paris : Hermann, 1903.

Han C., Vardoulakis I., "Plane-strain compression experiments on water-saturated finegrained sand", Géotechnique, vol. 47, n¹, 1991, p. 49-78.

Hassanizadeh M., Gray W., "General conservation equations for multi-phase systems: 1. Average procedure”, Advances in Water Resources, vol. 2, 1979, p. 131-144.

Hassanizadeh M., Gray W., "General conservation equations for multi-phase systems: 2. Mass, momenta, energy, and entropy equations", Advances in Water Resources, vol. 2, 1979, p. 191-208.

Hill R., "A general theory of uniqueness and stability in elastic-plastic solids", $J$. of Mechanics and Physics of Solids, vol. 5, 1958, p. 236-249.

Khoa H.D.V., Georgopoulos I.O., Darve F., Laouafa F., "Diffuse failure in geomaterials: Experiments and modelling", Computers and Geotechnics, vol. 33, n¹, 2006, p.1-14.

Kotronis P., Al Holo S., Bésuelle P., Chambon R., "Shear softening and localization. Modelling the evolution of the shear zone", Acta geotechnica, vol. 3, n², 2008, p. 85-97.

Lenoir N., Bornert M., Desrues J., Besuelle P., Viggiani G., "Volumetric digital image correlation applied to X-ray micro tomography images from triaxial compression tests on argillaceous rock", STRAIN, vol. 3, 2 2008, p. 193-205.

Lewis R.W., Schrefler B.A., The Finite Element Method in the Static and Dynamic Deformation and Consolidation of Porous Media, New York, Wiley, 2000.

Loret B., Prevost J., "Dynamic strain localization in fluid-saturated porous media", Journal of Engineering Mechanics, vol. 117, $\mathrm{n}^{\circ}$ 4, 1991, p. 907-922. 
Mandel J., "Condition de stabilité et postulat de Drucker", Proc. Of IUTAM symposium on rheology and soil mechanics, Grenoble, 1968, p. 58-67.

Mindlin R.D., "Micro-structure in linear elasticity", Arch. Ration. Mech. Anal., vol. 16, 1964, p. $51-78$.

Mokni M., Desrues J., "Strain localisation measurements in undrained plane-strain biaxial test on Hostun RF sand", Mechanics of cohesive-frictional materials, vol. 4, 1999, p. 419441.

Nuth M., Laloui L., "Effective stress concept in unsaturated soils: Clarification and validation of a unified framework", International Journal for Numerical and Analytical Methods in Geomechanics, vol. 32, n 7, 2008, p. 771-801.

Pijaudier-Cabot G., Bazant Z.P., "Non-local damage theory", Journal of Engineering Mechanics, ASCE, vol. 39, 1987, p. 1512-1533.

Peerlings R.H.J., De Borst R., Brekelmans W.A.M., De Vree J.H.P., "Gradient-enhanced damage for quasi-brittle materials", International Journal for Numerical Methods in Engineering, vol. 39, 1987, p. 1512-1533.

Rice J., "Theoretical and Applied Mechanics", North-Holland Publishing Company, chapter The localization of Plastic Deformation, 1976, p. 207-220.

Roger V., Desrues J., Viggiani G., "Experiments on strain localization in dense sand under isochoric conditions", in O.F.(ed.) Localization and Bifurcation Theory for Soils and Rocks, $4^{\text {th }}$ Workshop on Localization and Bifurcation Theory for soils and Rocks, Gifu (Japan) 28 septembre- 2 octobre 1997, Balkema, 1998, p. 239-248.

Rudnicki J, Rice J., "Conditions for the localisation of deformation in pressure sensitive dilatant materials", JMPS, vol. 23, 1975, p. 371-394.

Sieffert Y., Al Holo S., Chambon R., "Loss of uniqueness of solutions of the borehole problem modelled with enhanced media”, International Journal of Solids and Structures, to appear 2009, doi:10.1016/j.ijsolstr.2009.04.014.

Toupin R., "Elastic materials with couple-stresses", Archive for Rational Mechanics and Analysis, vol. 11, 1962, p. 385-414.

Vardoulakis I., Goldscheider M., Gudehus Q., "Formation of shear bands in sand bodies as a bifurcation problem", International Journal for Numerical and Analytical Methods in Geomechanics, vol. 2, 1978, p. 99-128.

Vardoulakis I., "Deformation of water-saturated sand: I. uniform undrained deformation and shear banding", Géotechnique, vol. 46, n 3, 1996a, p. 441-456.

Vardoulakis I., "Deformation of water-saturated sand: II. Effect of pore water flow and shear banding", Géotechnique, vol. 46, n 3, 1996b, p. 457-472.

Wang X.C., Modélisation numérique des problèmes avec localisation des déformations en bandes de cisaillement, Thèse de doctorat, Université de Liège, 1993.

Zhang H.W., Sanavia L., Schrefler B.A., "An internal length scale in dynamic strain localization of multiphase porous media", Mechanics of Cohesive-Frictional Materials, vol. $4, \mathrm{n}^{\circ} 5,1999$, p. 443-460. 
Zhang H.W., Schrefler B.A., "Uniqueness and localization analysis of elasto-plastic saturated porous media", International Journal for Numerical and Analytical Methods in Geomechanics, vol. 25, n 1, 2001, p. 29-48.

Zhang C, Schrefler B.A., "Particular aspects of internal length scales in strain localization analysis of multiphase porous materials", Computer Methods in Applied Mechanics and Engineering, vol. 193, 2004, p. 2867-2884. 